\title{
A 13-year-old female with shortness of breath and pleuritic chest pain
}

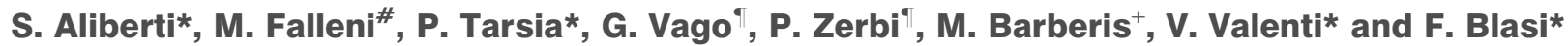

\section{CASE HISTORY}

A 13-yr-old Caucasian female was referred to the Dept of Paediatric Diseases (Havana, Cuba) with shortness of breath and pleuritic chest pain located in her right hemithorax. The pain had occurred the day before while doing physical activity and lasted $\sim 1 \mathrm{~h}$. The patient reported a 2-month history of recurrent episodes of pleuritic chest pain, which were either self-limited or relieved by paracetamol, as well as growing fatigue in the previous few weeks. Her past history was remarkable for a diagnosis of pneumonia after exposure to fumigation during a dengue haemorrhagic fever epidemic 2 yrs earlier, which was treated with steroids and antibiotics.

On admission, the patient had no weight loss, no fever or dyspnoea. Physical examination showed decreased breathing sounds in the inferior part of both hemithoraces. Cardiovascular, abdominal and neurological examination, as well as ear, nose and ophthalmologic investigations were

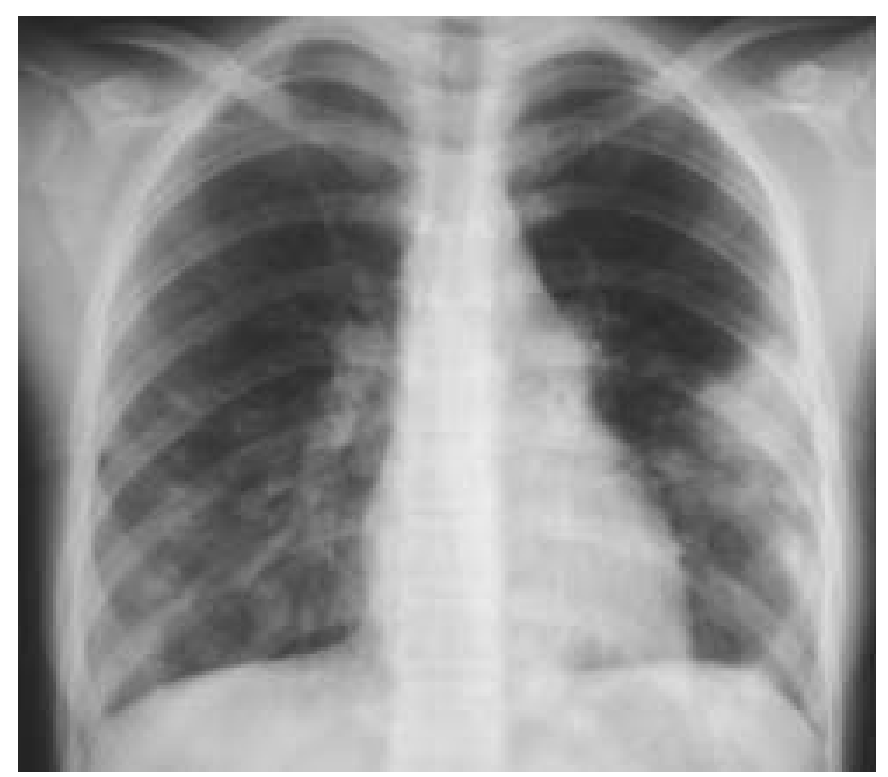

FIGURE 1. Chest radiograph obtained on October 24, 2003 (day 14 from onset). unremarkable. The chest radiograph showed bilateral pneumonia with moderate right-sided pleural effusion. The leukocyte count was $9.8 \times 10^{9}$ cells $\cdot \mathrm{L}^{-1}$ with $54 \%$ neutrophils and $32 \%$ lymphocytes. The erythrocyte sedimentation rate was $85 \mathrm{~mm} \cdot \mathrm{h}^{-1}$. Serum chemistry, renal and liver function tests were in the normal range. Blood cultures were negative. Antibiotic therapy with ceftriaxone and amikacin was started. During the treatment the patient reported 2 days of moderate fever and various episodes of left-sided pleuritic chest pain, which occurred especially during forceful diaphragm contractions (i.e. sneezing, laughing and coughing).

Following a short stay in the Cuban hospital, the patient was transferred to an Italian hospital (Como). Amikacin was discontinued and clarithromycin added. A new chest radiograph (fig. 1), 14 days after the previous one, and a computed tomography (CT) scan of the thorax were performed. Echocardiography and abdominal ultrasound were both negative. An increased value of the tumour marker CA-125 (220 U. $\mathrm{mL}^{-1}$, versus a normal value $<35 \mathrm{U} \cdot \mathrm{mL}^{-1}$ ) was found. Microbiological and blood testing workup results are shown in table 1 .

After 1 week, the patient was admitted to a hospital in Milan where a thoracic CT scan (fig. 2) and whole-body positron emission tomography (PET; fig. 3) were performed. The latter was obtained in order to identify any possible extrapulmonary focus of the disease. Further microbiological and blood testing is shown in table 1.

Pulmonary function tests showed a restrictive ventilatory pattern with a total lung capacity $76 \%$ of predicted, a vital capacity of $2.66 \mathrm{~L}$ (75\% pred), a forced expiratory volume in one second of $2.43 \mathrm{~L}$ ( $82 \%$ pred), a transfer factor of the lung for carbon monoxide of $6.52 \mathrm{mmol} \cdot \mathrm{min}^{-1} \cdot \mathrm{kPa}^{-1}(71 \%$ pred) and a transfer coefficient of the lung for carbon monoxide of $1.83 \mathrm{mmol} \cdot \mathrm{min}^{-1} \cdot \mathrm{kPa}^{-1} \cdot \mathrm{L}^{-1}(94 \%$ pred $)$.

Fibreoptic flexible bronchoscopy did not reveal any endobronchial lesion. Cultures of bronchoalveolar lavage (BAL) for bacteria, including Mycobacterium tuberculosis, and for fungi, and a search for malignant cells were negative. In order to obtain a diagnosis, lung biopsy by videothoracoscopy was performed and specimens from the left hemithorax were sent to a pathologist for evaluation (fig. 4).

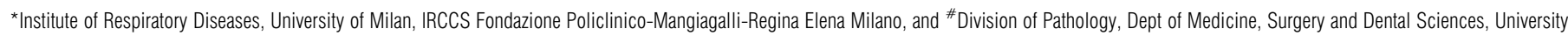

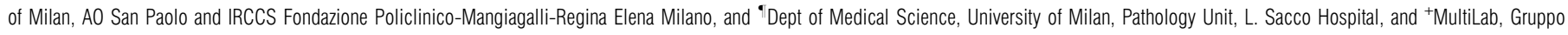
MultiMedica, Milan, Italy.

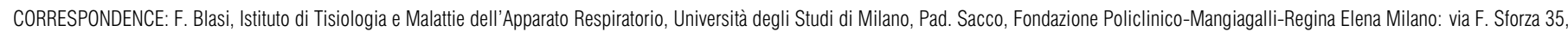
I-20122 Milan, Italy. Fax: 39 0250320628. E-mail: francesco.blasi@unimi.it 


\section{TABLE 1 Microbiological work-up and blood determinations}

\begin{tabular}{lll} 
Test Result & \\
\hline
\end{tabular}

\section{Admission to Como Hospital}

Erythrocyte sedimentation rate

C-reactive protein

$\lg \mathrm{M}$

Legionella pneumophila urinary antigen

Streptococcus pneumoniae urinary antigen

Mycoplasma pneumoniae IgM antibodies

Epstein-Barr serology

Cytomegalovirus serology

Toxoplasma gondii serology

Coxiella burnetii serology

Histoplasma serology (antigen and antibody)

Aspergillus serology (antigen and antibody)

Cryptococcus neoformans serology (antigen and antibody)

Coxsackievirus B1-6 serology

Tuberculin skin testing

Mycobacterium tuberculosis gastric aspirate and urine cultures

Parasite detection in the faeces

Chloride sweat test

Blood lymphocyte characterisation

\section{Admission to Milan Hospital}

Blood cultures

Chlamydia pneumoniae serology

Legionella pneumophila serology

Mycoplasma pneumoniae serology

Proteus serology

Salmonella typhi and paratyphi serology

Entoameba histolytica, Schistosoma haematobium, Toxocara canis,

Echinococcus serology

Rheumatoid factor and anti-ENA, anti-dsDNA, ANA, AMA, anti-SMA,

ANCA antibodies

Blood lymphocyte characterisation

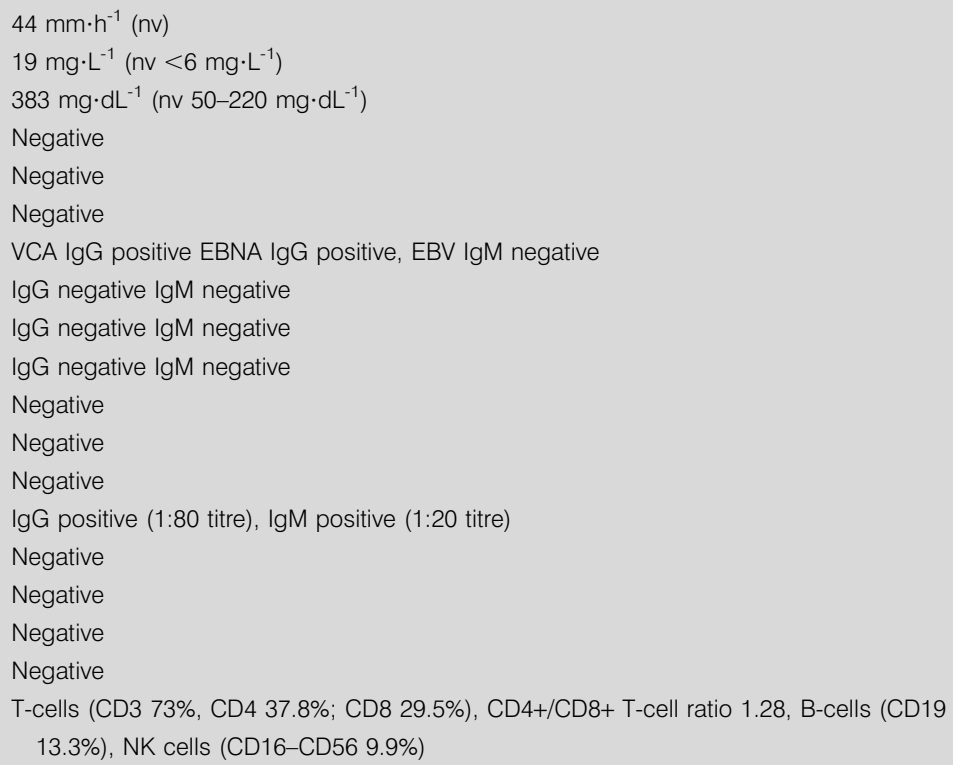

T-cells (CD5 80\%, CD3 77\%, CD4 46\%, CD8 31\%), CD4+/CD8+ ratio 1.47, B-cells (CD19 17\%, CD20 16\%, HLA-DR 18\%), NK cells (3.4\% (nv 12 $\pm 4 \%)$ )

nv: normal value; Ig: immunoglobulin; VCA: viral capsid antigen; EBNA: Epstein-Barr nuclear antigen; EBV: Epstein-Barr virus; NK: natural killer; ENA: extractable nuclear antigen; dsDNA: double-stranded DNA; ANA: anti-nuclear antibody; AMA: anti-mitochondrial antibody; SMA: smooth muscle actin; ANCA: anti-neutrophil cystoplasmic antibody; HLA-DR: human leukocyte antigen D-related. 


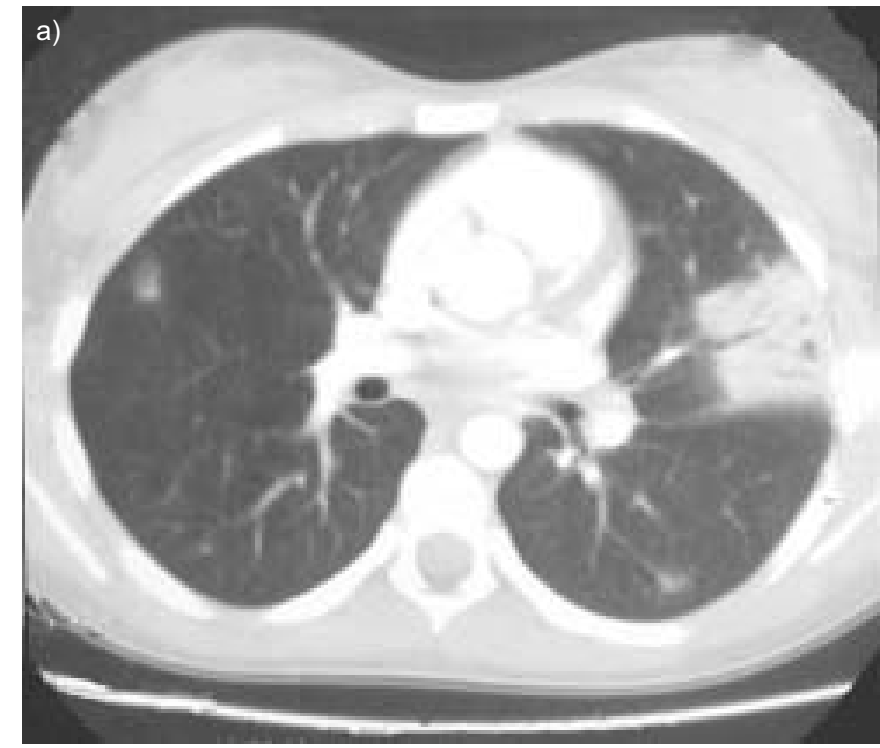

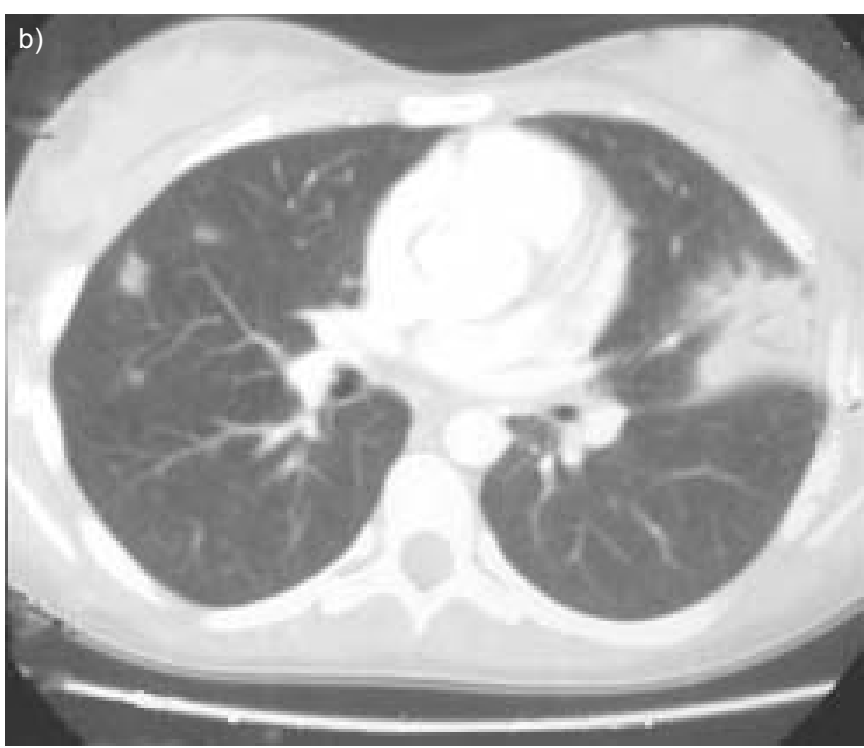

FIGURE 2. Two windows of the same chest computed tomography scan obtained on October 31, 2003 (day 21 from onset).

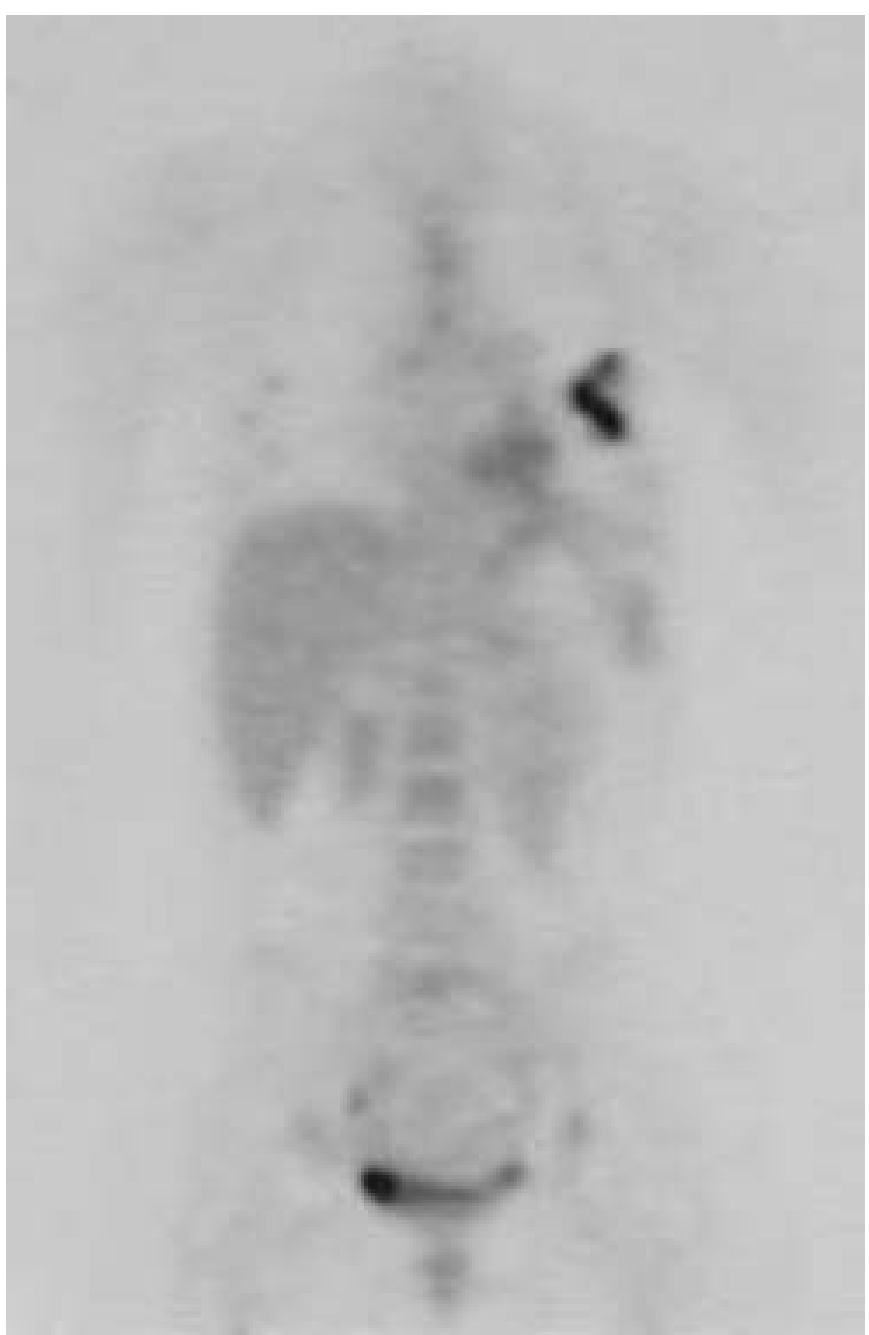

FIGURE 3. Total body positron emission tomography obtained on October 31, 2003 (day 21 from onset). 

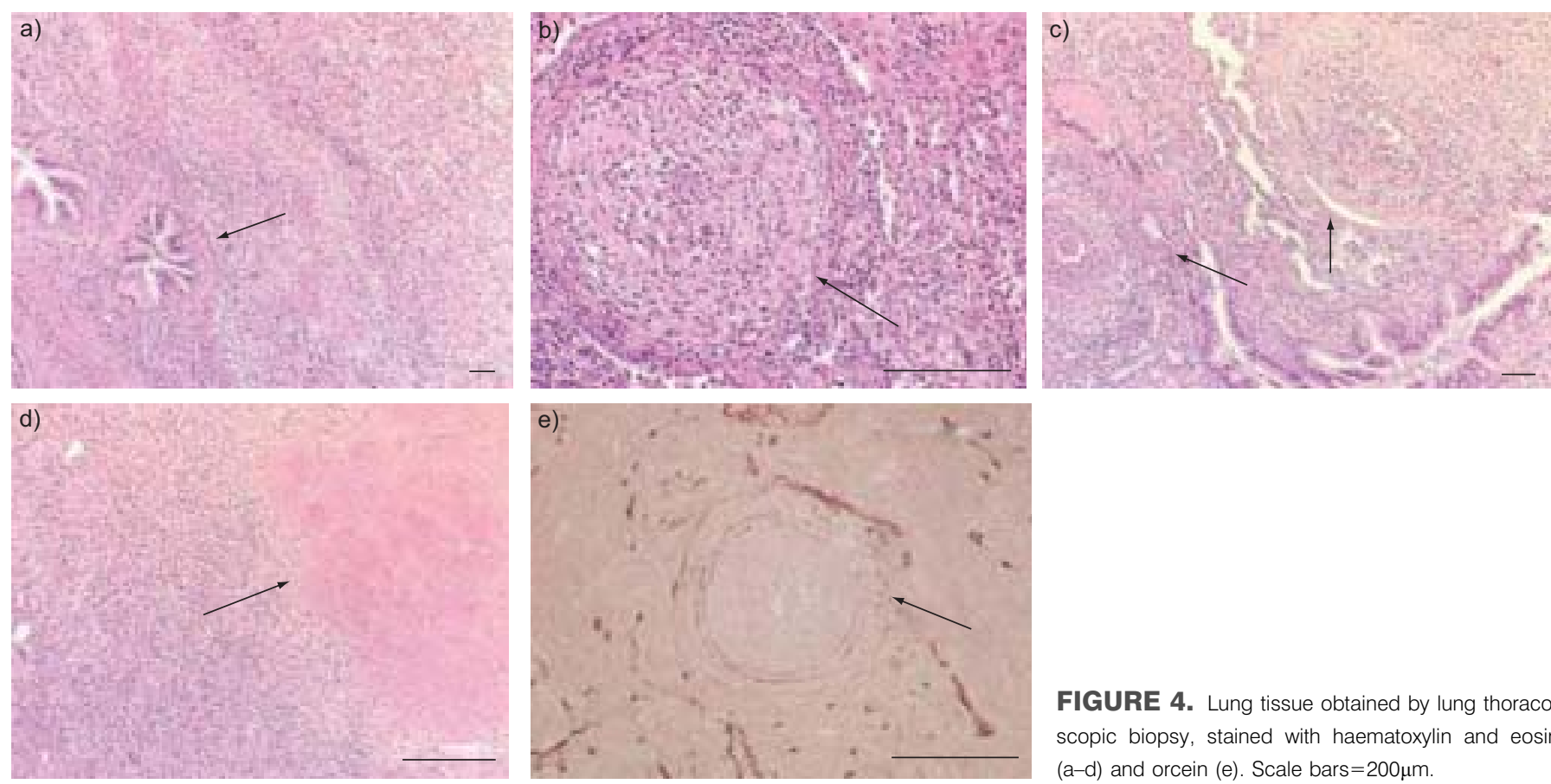

FIGURE 4. Lung tissue obtained by lung thoracoscopic biopsy, stained with haematoxylin and eosin (a-d) and orcein (e). Scale bars $=200 \mu \mathrm{m}$.

BEFORE TURNING THE PAGE, INTERPRET THE PATIENT'S HISTORY, CHEST RADIOGRAPH, COMPUTED TOMOGRAPHY SCANS, POSITRON EMISSION TOMOGRAPHY SCAN AND HISTOLOGY SLIDES, AND SUGGEST A DIAGNOSIS. 


\section{INTERPRETATION}

\section{Chest radiography}

The postero-anterior chest radiograph of the lungs (fig. 1) shows resolution of the right pleural effusion and the presence of a subpleural homogeneous lung opacity, with an irregular contour located in the left middle field. No hilar lymphadenopathy was detectable.

\section{Contrast-medium CT}

The contrast-medium CT scan (fig. 2) revealed a triangular lung infiltrate in the anterior segment of the left superior lobe between the apical segment of the inferior left lobe and the lingula with its base on the pleura.

A further area of lung consolidation was present in the left inferior lobe area and numerous subpleural nodular alterations could be seen bilaterally in the inferior and middle lobes distal to the peripheral vascular divisions. On both lung fields, particularly in the middle and lower areas, a large number of nodular lesions with no enhancement and a benign aspect was present.

\section{Whole-body PET}

Diffuse high-glucidic-metabolism lesions were apparent in both lungs (fig. 3). Considering the plurifocality of the lesions and the high glucose captation of active granulomas, the PET scans are compatible with a diagnosis of granulomas.

\section{Diagnostic considerations}

In the differential diagnosis of the present case, the current authors considered the following diagnoses: 1) immunederived granulomata (e.g. Wegener's granulomatosis); 2) infective granulomata; 3) metastatic lesions; and 4) cryptogenic organising pneumonia.

\section{Surgical specimens}

\section{Macroscopic features}

The lung subpleural specimen was $5 \mathrm{~cm}$ in greatest dimension, with yellowish nodules ranging $0.3-1.5 \mathrm{~cm}$ in diameter on the cut surface.

\section{Microscopic features}

Figure 4 shows the histological evaluation of the resected pulmonary lobe specimens. Figure 4a shows an intense peribronchial and vascular chronic inflammatory infiltrate consisting mainly of lymphocytes and plasma cells, and granulomas with multinucleated giant cells. Figure $4 \mathrm{~b}$ shows a noncaseating granuloma of tightly clustered epithelioid cells and interspersed multinucleated giant cells. Vasculitis with diffuse lymphocyte and plasma cell infiltrates and granulomas with multinucleated giant cells were seen in the peribronchial areas (fig. 4c). Large areas of necrosis (on the right-hand side of fig. $4 \mathrm{~d}$ ) were seen in the centre of nodules composed of confluent granulomas. Vasculitis with chronic inflammatory infiltrates of plasma cells and lymphocytes and/or granulomatous reaction was mainly observed in areas with intense granulomatous reaction and is easily evident with special stains highlighting the wall of the vessels (fig. 4e).

Possible rare filamentous, Gram-positive and weakly periodic acid-Schiff positive, alcohol- and acid-resistant microorganisms were found in some of the sections. PCR assays were negative for both typical and atypical mycobacteria and Actinomyces. Thus, the organisms were considered innocent bystanders.

\section{Diagnosis: Necrotising sarcoid granulomatosis.}

\section{CLINICAL COURSE}

The patient was discharged with no therapy and remained asymptomatic. A CT scan taken 3 months later showed complete resolution of the opacities located in the middle and lower fields of both hemithoraces. There was a reduction in the size of the subpleural opacity in the left lower lobe. The erythrocyte sedimentation rate was $14 \mathrm{~mm}$ (after $1 \mathrm{~h}$ ) and $24 \mathrm{~mm}$ (after $2 \mathrm{~h}$ ); leukocyte count and C-reactive protein values were in the normal range.

\section{DISCUSSION}

Necrotising sarcoid granulomatosis (NSG), first described by LIEBOW in 1973 [1], is a rare form of pulmonary vasculitis and granulomatosis that mainly occurs in middle-aged females (40-50 yrs) [2-4]. NSG in childhood, to the current authors' knowledge, has been described in literature only four times and in only two of these cases $[5,6]$ was the pulmonary involvement isolated, as in the case described here. In the other two cases $[7,8]$ neurogical and ophthalmological lesions were encountered as well.

NSG is currently included in the group of pulmonary angiitis and granulomatosis, along with four other distinct clinical syndromes: Wegener's granulomatosis, lymphomatoid granulomatosis, bronchocentric granulomatosis, and allergic angiitis and granulomatosis (Churg-Strauss syndrome). The aetiology and pathogenesis of NSG is still unknown. Diagnosis can be reached only by pathological investigation [2, 9], through identification of sarcoid-like granulomas (fig. 4b), granulomatous vasculitis (fig. 4c) and necrosis (fig. 4d).

Cough, dyspnoea and chest pain are nonspecific pulmonary symptoms that may be present, either with or without systemic involvement, such as fever, weight loss and weakness [4]. However, $25 \%$ of patients are asymptomatic. The presentation of NSG in the 13-yr-old female was remarkable for several reasons, including the previous history of recurrent pleuritic chest pain and the onset of an "atypical" bilateral pneumonia. A large discrepancy was in fact present between the mild clinical presentation and the extensive radiological involvement. The recurrence of chest pain episodes and the occurrence of fever during antibiotic treatment prompted thorough diagnostic investigations to identify possible underlying diseases. This strategy was later borne out when the pneumonia was resolved but underlying chest radiography and CT opacities persisted.

The typical radiographic findings with NSG are single or multiple lung opacities [10], with common involvement of the pleura $[5,11]$, as was observed in this case (figs $2 a$ and b). Lymphadenopathy in NSG is reported in $10-65 \%$ of cases [11, 12], whereas previous reports in adult [13] and paediatric patients [5] found no hilar or mediastinic lymph node enlargement, as occurred in the current case. No typical laboratory and radiographic/functional findings can be of help in diagnosing NSG. The erythrocyte sedimentation rate was elevated in this patient, a finding present in some [9], but not 
all papers in the literature [6]. Pulmonary function tests showed a restrictive pattern, as in other previous cases [5, $11]$, but this finding is not universal $[4,6]$. The CD4+/CD8+ ratio in the peripheral blood of the current patient was normal; however, in a previous case [6] this value was reported to be reduced early in the disease, but later normalised. The CD4+/ CD8+ T-cell ratio in the BAL was normal too. Serum levels of immunoglobulin $\mathrm{M}$ were increased in the current patient, as in the case described of another 12-yr-old female with NSG [8].

Immunopathological findings are strikingly common between NSG and sarcoidosis. Whether NSG should be considered a distinct entity $[14,15]$ or a subform of sarcoidosis [3, 12, 16-18] with a similar immunopathological basis [19] remains, to date, an open question. Nonnecrotising granulomas and angiitis may be detected both in NSG and sarcoidosis, particularly in the less severe forms of the latter. In addition, $5 \%$ of patients with sarcoidosis show histological characteristics typical of NSG [3, 20]. Hilar lymphadenopathy and extrapulmonary manifestations are more frequent in sarcoidosis [11, 15], as is the presence of an elevated serum angiotensin-converting enzyme (ACE) level. In the present case, ACE could not be assessed, but in most of the previously reported cases of NSG $[4-6,13]$ it was in the the normal range. In terms of outcome, both diseases generally show a benign clinical course with a favourable prognosis.

Differential diagnoses include tuberculosis, Wegener's granulomatosis, lymphomatoid granulomatosis, neoplasms, hypersensitivity pneumonitis or Churg-Strauss syndrome. Roughly $20 \%$ of Wegener's granulomatosis cases show sarcoid-like granulomata; in the current case, this possibility was ruled out by the absence of anti-neutrophil cytoplasmic autoantibodies and the subsequent benign clinical course.

Cultural and serological studies ruled out most common infectious diagnoses. Antibodies to Mycoplasma pneumoniae were detected, suggesting this agent as the cause of the bilateral pneumonia in the current patient. Evidence of Chlamydia pneumoniae infection has previously been reported in a 14-yr-old female NSG patient [5]. On this basis some authors have hypothesised that NSG could be a form of hypersensitivity pneumonitis [9]. Other authors found Aspergillus antigen upon immunohistochemical staining of granulomas [9]. Although so far undetected in NSG, immune complexes may be involved in its pathogenesis [21].

The clinical course of NSG is usually benign [13,16], and in most cases treatment is not required. The patient reported was discharged without any treatment and 3 months later complete clinical and radiographic resolution of the disease was witnessed. A favourable outcome was also seen in an 8-yrold patient whose symptoms and radiographic findings disappeared in the absence of therapy [6]. Experience in terms of therapy is unfortunately still limited. Data from adults suggest that corticosteroids and immunosuppressive therapy should be restricted to patients with progressive or severe NSG, particularly in cases with central nervous system involvement $[4,7,9,22,23]$.

In conclusion, the current authors present the clinical features of a 13-yr-old child with a diagnosis of necrotising sarcoid granulomatosis. The disease showed spontaneous remission in the absence of treatment. In the differential diagnosis of lung granulomatous lesions, necrotising sarcoid granulomatosis should be kept in mind in paediatric patients in addition to adults.

\section{REFERENCES}

1 Liebow AA. The J. Burns Amberson lecture: pulmonary angiitis and granulomatosis. Am Rev Respir Dis 1973; 1089: $1-17$.

2 Le Gall F, Loeuillet L, Deleval P, Thoreux PH, Desrues B, Ramee MP. Necrotizing sarcoid granulomatosis with and without extrapulmonary involvement. Path Res Pract 1996; 192: 306-313.

3 Churg A. Pulmonary angiitis and granulomatosis revisited. Hum Pathol 1983; 14: 868-883.

4 Quaden C, Tillie-Leblond I, Delobbe A, et al. Necrotising sarcoid granulomatosis: clinical, functional, endoscopical and radiographical evaluations. Eur Respir J 2005; 26: 778-785.

5 Tauber E, Wojnarowski C, Horcher E, Dekan G, Frischer T. Necrotizing sarcoid granulomatosis in a 14-yr-old female. Eur Respir J 1999; 13: 703-705.

6 Heinrich D, Gordjani N, Trusen A, Marx A, Hebestreit H. Necrotizing sarcoid granulomatosis: a rarity in childhood. Pediatr Pulmonol 2003; 35: 407-411.

7 Beach RC, Corrin B, Scopes JW, Graham E. Necrotizing sarcoid granulomatosis with neurologic lesions in a child. $J$ Pediatr 1980; 97: 950-953.

8 Singh N, Cole S, Krause PJ, Conway M, Garcia L. Necrotizing sarcoid granulomatosis with extrapulmonary involvement. Am Rev Respir Dis 1981; 124: 189-192.

9 Koss MN, Hochholzer L, Feigin DS, Garancis JC, Ward PA. Necrotizing sarcoid-like granulomatis; clinical, pathological and immunopathologic findings. Human Pathol 1980; 11: 510-519.

10 Niimi H, Hartmann TE, Müller NL. Necrotising sarcoid granulomatosis: computed tomography and pathologic findings. J Comput Assist Tomogr 1995; 19: 920-923.

11 Chittock DR, Joseph MG, Paterson NA, McFadden RG. Necrotizing sarcoid granulomatosis with pleural involvement. Chest 1994; 106: 672-676.

12 Churg A, Carrington CB, Gupta R. Necrotizing sarcoid granulomatosis. Chest 1979; 76: 406-413.

13 van Esser JW, van Belle AF, Lamers RJ, Thunnissen FB, Wesseling GJ, Wouters EF. A young woman with cough and chest pain. Eur Respir J 1995; 8: 664-666.

14 Saldana MJ. Necrotizing sarcoid granulomatosis: clinicopathologic observations in 24 patients [abstract]. Lab Invest 1978; 38: 364.

15 Rolfes DB, Weiss MA, Sanders MA. Necrotizing sarcoid granuomatosis with suppurative features. I Clin Pathol 1984; 82: 602-607.

16 Luce JM. Vasculitis, primary pulmonary hypertension, and arteriovenous fistulas. In: Murray JF, Nadel JA, eds. Textbook of Respiratory Medicine. Philadelphia, WB Saunders, 1998; pp. 1328-1355.

17 Gibbs AR, Anderson A. Pulmonary vasculitis and eosinophilic pneumonia. In: Brewis RAL, Gibson GJ, Geddes DM, eds. Respiratory Medicine. London, Bailliere Tindall, 1990; pp. 1165-1182. 
18 Bouman KP, Slabbynck H, Cuykens JJ, Galdermans D, Coolen D, Kockx M. Necrotizing sarcoid granulomatosis with uveitis: a variant of sarcoidosis? Acta Clin Belg 1997; 52: 367-370.

19 Spiteri MA, Gledhill A, Campbell D, Clarke SW. Necrotizing sarcoid granulomatosis. Br J Dis Chest 1987; 81: 70-75.

20 Rosen Y, Moon S, Huang C, Gourin A, Lyons A. Granulomatous pulmonary angiitis in sarcoidosis. Arch Pathol Lab Med 1977; 101: 170-174.
21 Leavitt RY, Fauci AS. Pulmonary vasculitis. Am Rev Respir Dis 1986; 134: 149-166.

22 Radenne F, Tillie-Leblond I, Maurage CA, Remy-Jardin M, Wallaert B, Tonnel AB. Sjögren's syndrome and necrotizing sarcoid-like granulomatosis. Rev Mal Respir 1999; 16: 554-557.

23 Gibbs AR, Williams WJ, Kelland D. Necrotizing sarcoidal granulomatosis: a problem of identity. A study of seven cases. Sarcoidosis 1987; 4: 94-100. 